\title{
Pectin-Based Biodegradable Hydrogels with Potential Biomedical Applications as Drug Delivery Systems
}

\section{Mohammad Sadeghi}

Department of Chemistry, Science Faculty, Islamic Azad University, Arak Branch, Arak, Iran

Email: m-sadeghi@iau-arak.ac.ir

Received October $28^{\text {th }} 2010$; November $23^{\text {rd }} 2010$; November $30^{\text {th }} 2010$.

\begin{abstract}
In this work, synthesis and swelling behavior of a superabsorbent hydrogel based on Pectin $(P c)$ and polyacrylonitrile (PAN) were investigated. A mechanism for hydrogel formation was proposed and the structure of the product was established using FTIR spectroscopy. The Pc-poly (sodium acrylate-co-acrylamide) hydrogel exhibited a pH-responsive swelling-deswelling behavior at pH's 2 and 8. This on-off switching behavior provides the hydrogel with the potential to control delivery of bioactive agents. Release profiles of ibuprofen (IBU), a poor water-soluble drug, from the hydrogels were studied under both simulated gastric and intestinal $\mathrm{pH}$ conditions.
\end{abstract}

Keywords: Pectin, Polyacrylonitrile, Hydrogel, Ibuprofen, Drug Delivery

\section{Introduction}

Pectin is a naturally occurring biopolymer that is finding increasing applications in the pharmaceutical and biotechnology industry. It has been used successfully for many years in the food and beverage industry as a thickening agent, a gelling agent and a colloidal stabilizer. Pectin also has several unique properties that have enabled it to be used as a matrix for the entrapment and/or delivery of a variety of drugs, proteins and cells. Furthermore, crosslinked polymers from Pectin can form hydrogels that are able to absorb and retain hundreds of times their weight of water and are known as superabsorbents [1]. The properties of these hydrogels have attracted the attention of many researchers and technologists and have found wide-spread applications in many fields, such as drug delivery systems, agriculture, separation processes [2-5]. The combination of the hydrophilic acrylic polymer properties with the biodegradable character of Pectin based blends, will lead to interesting hydrogels with potential applications as biomaterials exhibiting different properties depending on the composition and on the type of interactions within the network, attending to chemical crosslinking and hydrogen bonding interactions [6]. This work deals with the development of new biodegradable hydrogels developed by the polymerization of $\mathrm{AN}$ and some formulations with sodium hydroxide as crosslinker, in the presence of Pectin.

\section{Experimental}

\subsection{Hydrogel Preparation}

A one step preparative method was used for synthesis of Pc-poly(sodium acrylate-co-acrylamide)hydrogel, Pc-poly (NaAA-co-AAm), hydrogel. Pectin (1.33 g) was added to $35 \mathrm{~mL}$ of doubly distilled water in a three-neck reactor equipped with a mechanical stirrer (Heidolph RZR 2021, three blade propeller type). The reactor was immersed in a thermostated water bath. After complete dissolution of the Pectin, sodium hydroxide (10.0 wt \%) was added to the Pectin solution at desired temperature (alkalization temperature, $80^{\circ} \mathrm{C}$ ). The mixture was allowed to stir for certain times (alkalization times, $120 \mathrm{~min}$ ). The various amount of polyacrylonitrile $(1.0 \mathrm{~g})$ was dispersed in the reaction mixture to saponify for certain times and temperatures (alkaline time and temperature). During the saponification $\mathrm{NH}_{3}$ gas was evolved and a color change from red to light yellow. This discoloration was an indication of the reaction completion. The pasty mixture was allowed to cool to room temperature and neutralized to $\mathrm{pH} 8.0$ by addition of $10 \mathrm{wt} \%$ aqueous acetic acid solution. Then the gelled product was scissored to small 
pieces and poured in ethanol $(200 \mathrm{~mL})$ to dewater for $5 \mathrm{~h}$. The hardened particles were filtered and dried in oven $\left(50^{\circ} \mathrm{C}, 10 \mathrm{~h}\right)$. After grinding, the powdered superabsorbent hydrogel was stored away from moisture, heat and light.

\subsection{Drug Loading Efficiency and In Vitro Drug Release}

Powdered samples $(1 \mathrm{~g} \pm 0.0001)$, with average particle sizes between 40-60 mesh (250-420 $\mu \mathrm{m})$, were accurately weighted and immersed in an alkaline solution of ibuprofen (IBU, $0.54 \mathrm{~g}$ dissolved in $50 \mathrm{~mL}$ distilled water) at $0^{\circ} \mathrm{C}$ for $25 \mathrm{~h}$. The swollen hydrogels loaded with drug were placed in a vacuum oven and dried under vacuum at $37^{\circ} \mathrm{C}$. The loading amount of drug in the hydrogels was calculated from the decrease in the concentration of the IBU solution which was determined using a UV spectrophotometer (UV-1201, Shimadzu, Kyoto, Japan). The loading efficiency of the Pectin-based hydrogels was calculated as the ratio of the final to the initial IBU concentration.

In vitro release was carried out in duplicate by incubating $0.01 \pm 0.0001 \mathrm{~g}$ of the IBU-loaded hydrogels into a cellophane membrane dialysis bag ( $\mathrm{D}_{9402}$, SIGMA-ALDRICH) in $50 \mathrm{~mL}$ of buffer solution (either $\mathrm{pH} 1.2$ or 7.4 ) at $37^{\circ} \mathrm{C}$. At specific time intervals, $1 \mathrm{~mL}$ aliquots of sample was withdrawn and after suitable dilution the concentration of drug released was measured by UV spectrophotometer. The drug release percent was calculated twice using the following equation:

$$
\text { Released drug }(\%)=\mathrm{R}_{\mathrm{t}} / \mathrm{L} \times 100
$$

where $L$ and $R_{t}$ represent the initial amount of drug loaded and the final amount of drug released at time $t$.

\section{Results and Discussion}

\subsection{Mechanism of Hydrogel Formation}

A general reaction mechanism for Pc-poly(NaAA-coAAm) hydrogel formation is shown in Scheme 1. In the first step, Pectin hydroxyl groups were converted to corresponding alkoxides using sodium hydroxide. These macroalkoxides initiated a crosslinking reaction between adjacent polyacrylonitrile pendant chains forming naphthyridine cyclic structures (including imine, $-\mathrm{C}=\mathrm{N}-$, conjugated bonds with deep red color). The intermediate was then hydrolyzed using residual sodium hydroxide aqueous solution to produce hydrophilic carboxamide and carboxylate groups (Scheme 1) with a resulting color change from red to light yellow. This sharp color change was used as an indication to halt the alkaline treatment. However, incompletely hydrolyzed structures may also give rise to a few crosslinking points result in a loosely crosslinked network.

Infrared spectroscopy was carried out to confirm the chemical structure of the materials obtained. The FTIR spectra of Pc-PAN physical mixture and the resulted hydrogel, Pc-poly(NaAA-co-AAm) are shown in Figure 1. The band observed at $2242 \mathrm{~cm}^{-1}$ was attributed to stretching of cyanide (Figure 1a). The Pectin backbone with side chains with carboxamide and carboxylate functional groups were identified by three new peaks at 1407, 1556, and $1675 \mathrm{~cm}^{-1}$ (Figure 1b).

These peaks were attributed to $\mathrm{C}=\mathrm{O}$ stretching in carboxamide functional groups and symmetric and asymmetric stretching modes of carboxylate groups, respecttively [7].

The in situ reaction was conducted with alkaline hydrolysis in absence of the polysaccharide. The product obtained was soluble, indicating that crosslinks were not being formed without Pectin. This substantiated the fact that Pectin hydroxyls are involved in the crosslinking process leading to the formation of hydrogels.

\subsection{PH-Reversibility for Pc-Poly (NaAA-co-AAm) Hydrogel}

Since the hydrogels show different swelling behavior at various $\mathrm{pHs}$, we investigated their $\mathrm{pH}$-reversibility in solutions buffered at $\mathrm{pH} 2.0$ and 8.0. A stepwise reproducible in swelling change of the hydrogel at $25^{\circ} \mathrm{C}$ with alternating pH between 2.0 and 8.0 is seen in Figure 2 .

At $\mathrm{pH} 8.0$, the hydrogel swelled up to $157 \mathrm{~g} / \mathrm{g}$ due to anion-anion repulsive electrostatic forces, while, at $\mathrm{pH}$ 2.0 , it shrunk within a few minutes due to protonation of carboxylate groups. This sharp swelling-deswelling behavior of the hydrogels makes them suitable candidates for controlled drug delivery systems. Such on-off switching behavior as reversible swelling and deswelling has been reported for other ionic hydrogels [8-11].

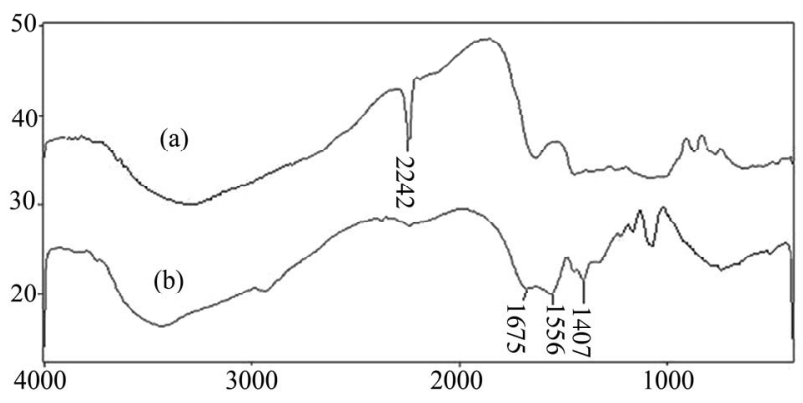

Transmittance/Wavenumber $\left(\mathrm{cm}^{-1}\right)$

Figure 1. FTIR spectra of (a) the physical mixture of Pectin and PAN and (b) the crosslinked Pc-poly (NaAA-co-AAm) hydrogel. 


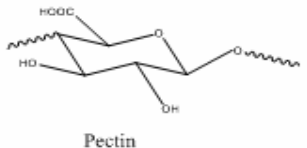

$1 \mathrm{Ce}^{4+}$

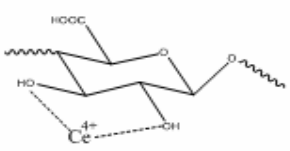

$\mid \begin{aligned} & -\mathrm{Ce}^{3+} \\ & -\mathrm{H}^{+}\end{aligned}$

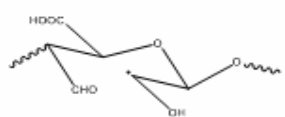

$\downarrow$
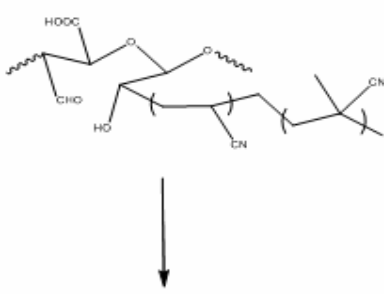

Pectin-g-PAN copolymer

(a)

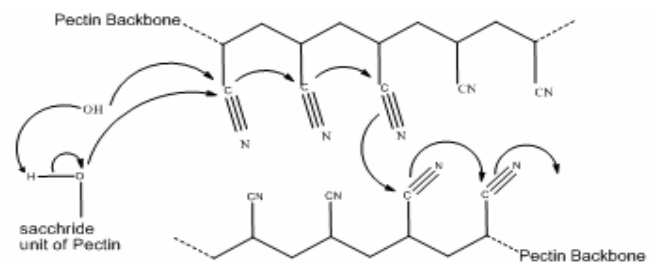

(Pectin-g-PAN copolymer)

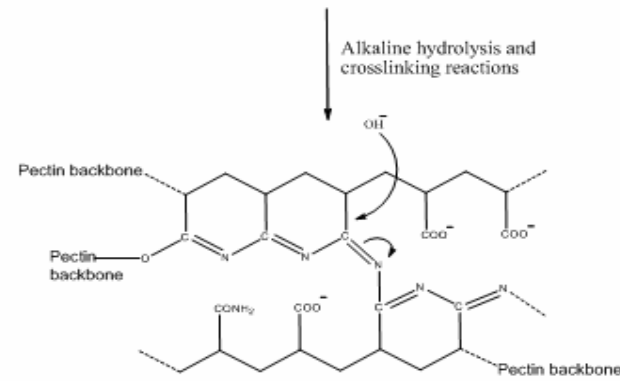

(deep orange- red )
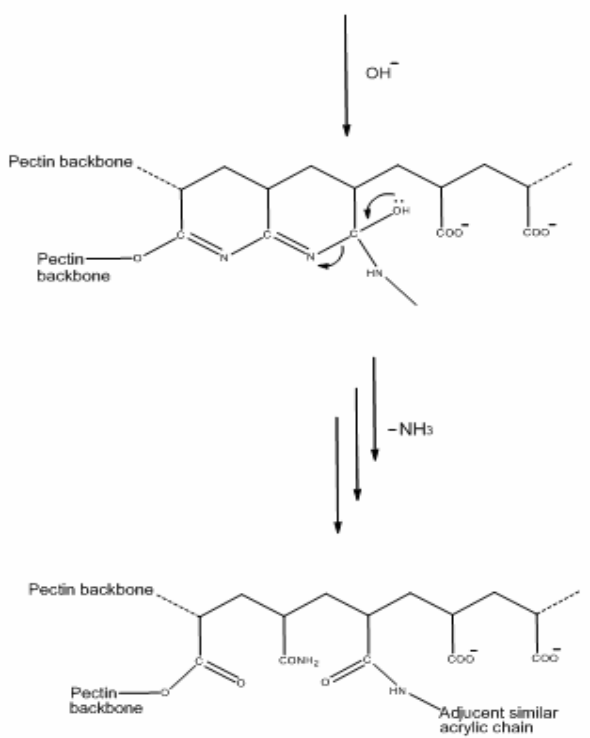

H- Pectin-g-PAN copolymer

(light yellow)

(b)

Scheme 1. Proposed mechanism for crosslinking during the hydrolysis of nitrile groups of the Pc-PAN mixture to produce the Pc-poly (NaAA-co-AAm) hydrogel.

\subsection{In vitro IBU Release in the Simulated Human Gastrointestinal System}

To determine the potential application of Pectin-based superabsorbent containing a pharmaceutically active compound, we have investigated the drug release behavior IBU form this system under physiological conditions. The percent of released drug from the polymeric carriers as a function of time is shown in Figure 3.
The concentration of IBU released at selected time intervals was determined by UV spectrophotometer. The IBU-loaded hydrogels with high degrees of drug loading $(>80 \%)$ were prepared by the swelling-diffusion method. The amount of IBU released in a specified time from the Pectin-based hydrogel decreased as the $\mathrm{pH}$ of the dissolution medium was lowered, indicating better release in a medium with a $\mathrm{pH}$ much higher than that of the stomach.

At low $\mathrm{pH}$ values, electrostatic repulsion between the 


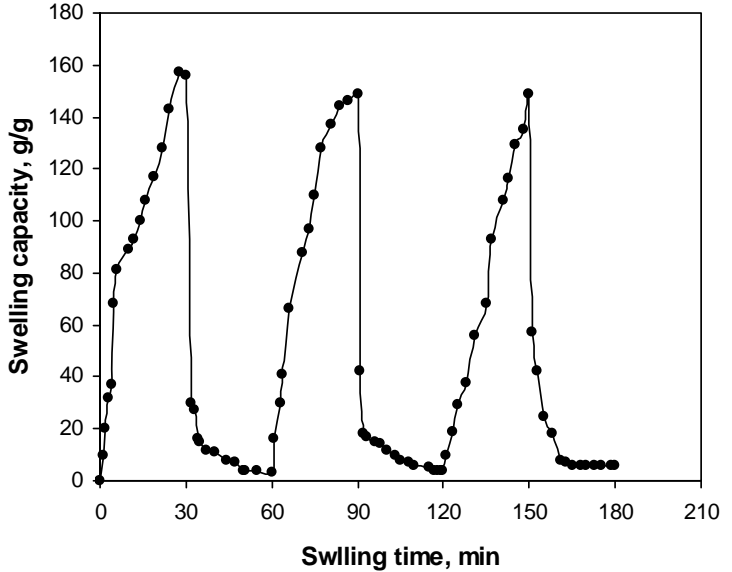

Figure 2. On-off switching behavior as reversible pulsatile swelling (pH 8.0) and deswelling (pH 2.0) of Pc-poly(NaAA-co-AAm) hydrogel. The time interval between the $\mathrm{pH}$ changes was $30 \mathrm{~min}$.

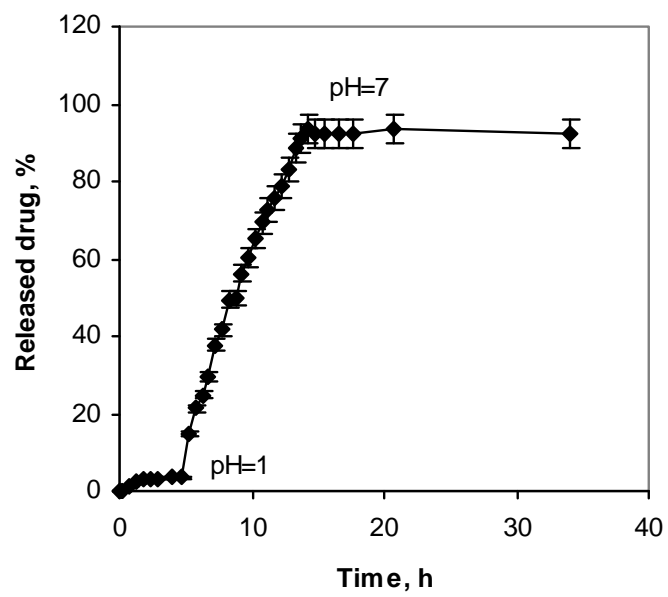

Figure 3. Release of IBU from hydrogel carrier as a function of time and $\mathrm{pH}$ at $37^{\circ} \mathrm{C}$.

carboxylic acid groups of backbone is low, thus decreases gel swelling and minimizes release of IBU via diffusion. However, in alkaline media the presence of $\mathrm{OH}^{-}$increases the electrostatic repulsion between carboxylate groups, thus increases the gels swelling degree and so the release of IBU was increased [12-13]. The amounts of the loaded drug in superabsorbent hydrogels was significantly affected by the loading time (Figure 4). With increasing loading time, the amount of drug loaded is initially increased and then begins to level off.

\section{Conclusion}

The superabsorbent hydrogel, Pc-poly(NaAA-co-AAm), was synthesized through alkaline hydrolysis of Pc-PAN physical mixture. The reaction of Pectin alkoxide anions with nitrile groups of polyacrylonitrile, formed crosslinks

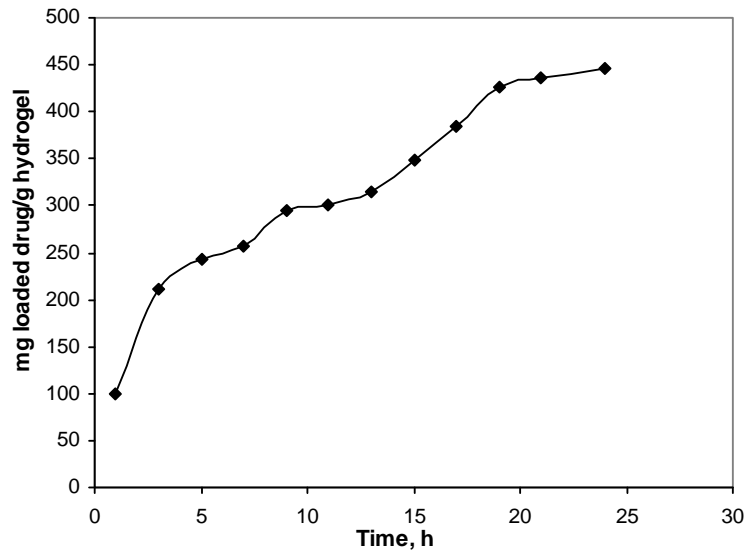

Figure 4. The dependency of the drug loading amount to the loading time.

producing a three-dimensional network. No toxics material were used in the synthesis, this practical approach may be preferred to as a relatively "green process". This one-step preparative method conducted under normal atmospheric conditions in a short period of time. The dark red-yellow color change provided a visual indication for the reactions progress. The superabsorbent hydrogels exhibited high sensitivity to $\mathrm{pH}$, so that, the reversible swelling-deswelling behavior in solutions with acidic and basic $\mathrm{pH}$, contributes to the suitability of these hydrogels as candidates for controlled drug delivery systems. In vitro drug-release studies in different buffer solutions showed that the most important parameter affecting the drug-release behavior of hydrogels is the $\mathrm{pH}$ of the solution. The release value of Ibuprofen from hydrogels at $\mathrm{pH} 7.4$ was higher than that at $\mathrm{pH} 1.2$ due to the electrostatic repulsion between carboxylate groups.

\section{Acknowledgements}

The author thanks the Research center of Islamic Azad University of Arak.

\section{REFERENCES}

[1] F. L. Buchholz and A. T. Graham "Modern Superabsorbent Polymer Technology," Wiley, New York, 1997.

[2] Po R. "Water-absorbent Polymers, A Patent Survey," Journal of Macromolecular Science, Reviews in Macromolecular Chemistry \& Physics, C34, 1994, pp. 607-661.

[3] J. Kost, "Encyclopedia of Controlled Drug Delivery," E. Mathiowitz, Ed. Wiley, New York, 1999.

[4] A. S. Hoffman, "Polymeric Materials Encyclopedia," J. C. Salamone; Ed., CRC Press, Boca Raton, FL. 5, 1996.

[5] N. A. Peppas and A. G. Mikes, "Hydrogels in Medicine and Pharmacy," CRC Press Inc., Boca Raton, Florida.1, 27(1986).

[6] P. J. Flory, "Principles of polymer chemistry," Ithaca, NY: 
Cornell University Press, 1953.

[7] R. M. Silverstein and F. X. Webster, "Spectrometric Identification of Organic Compounds," 6th Edition, Wiley: New York, 1998.

[8] A. Pourjavadi, M. Sadeghi and H. Hosseinzadeh (2004). Polymers for Advanced Technologies, 15: pp. 645-653.

[9] A. M. Lowman and N. A. Peppas, "Hydrogels", In: E. Mathiowitz, Ed. Encyclopedia of Controlled Drug Delivery, Wiley, New York, 1999, p. 139.
[10] Z. Mohamadnia, M. J. Zohuriaan-Mehr, K. Kabiri, A. Jamshidi and H. Mobedi, Journal of Bioactive and Compatible Polymers, Vol. 22, 2007, pp. 342.

[11] L. H. Gan, G. R. Deen, Y. Y. Gan and K. C. Tam, European Polymer Journal, Vol. 37, 2001, pp. 1473-1478.

[12] M. Mahkam, L. Doostie and S. O. R. Siadat (2006). Inflammo Pharmacology Vol. 14, 2006, pp. 72-75.

[13] M. Mahkam and M. Allahverdipoor, Drug Targeting, Vol. 12, 2004, pp. 151-156. 